\title{
The effects of instructions and an engaging visual task on habituation to loud tones: An evaluation of an alternative to the traditional habituation paradigm
}

\author{
WILLIAM G. IACONO \\ University of British Columbia, Vancouver, B.C., Canada \\ and \\ DAVID T. LYKKEN \\ University of Minnesota, Minneapolis, Minnesota
}

\begin{abstract}
The effects of ambiguous instructions and instructions to specifically ignore or attend to stimuli on electrodermal habituation were examined in 88 volunteers who were evenly divided among four groups. The subjects were exposed to 17 105-dB tones. Subjects in three of the groups watched a videotape of an old silent movie. Those in the "Ignore" group were exhorted to immerse themselves in the movie and ignore the tones. "Attend" subjects were told to count the tones and be certain that they all sounded the same. In the two neutral groups, only one of which saw the movie, subjects were told simply that they would hear some tones. "Ignore" subjects responded less often, habituated at a faster rate, and rated the tones as less loud than did subjects in the other groups. The results confirmed our previous findings that ignore instructions coupled with a distracting task reduce electrodermal reactivity to loud task-irrelevant stimuli.
\end{abstract}

A great deal of research has focused on the stimulus features and subject characteristics that affect habituation of the orienting response (e.g., Kimmel, van Olst, \& Orlebeke, 1979). How the task is presented to the subject can also be expected to affect the habituation process, but relatively little attention has been paid to the influence of this factor. In the typical study, participants are told that they will be exposed to some stimuli (e.g., tones), and, other than being instructed not to move about, they have no particular task. Such instructions essentially invite subjects to decide for themselves how meaningful the stimuli are, and they can be expected to lead to individual differences in reactivity that might affect habituation rate. The fact that this sort of ambiguous habituation instruction is routinely used in psychopathology and personality studies may be partly responsible for the weak and inconsistent relationships reported in these investigations (Iacono \& Lykken, 1979; see Bernstein, Frith, Gruzelier, Straube, Venables, \& Zahn, 1982, Iacono, Lykken, Peloquin, Lumry, Valentine, \& Tuason, 1983, and O'Gorman,

We would like to thank Kevin Haroian and Tim Brandow for their assistance with data collection and analysis. Address requests for reprints to: William G. Iacono, Department of Psychology, University of British Columbia, 154-2053 Main Mall, Vancouver, B.C., Canada V6T 1 Y7.
1977, for reviews of the psychopathological and personality literature pertaining to habituation).

We have argued that because habituation involves a capacity to tune out and stop responding to irrelevant stimuli, a more sensible procedure for measuring individual differences in habituation would be to tell subjects we were measuring their ability to stop reacting to a meaningless stimulus (Iacono \& Lykken, 1979, 1983). Since instructing subjects to ignore the only stimuli to which they are exposed may impart signal value to the stimuli, we have maintained that to ensure stimulus irrelevance, subjects should be provided with a distracting task that would enable them to focus their attention away from the stimuli.

The research literature regarding the effects of instructions on the electrodermal orienting response and habituation has generated conflicting findings. Instructions to ignore the experimental stimuli have been associated with both enhanced (Kohlenberg, 1970) and diminished (Tizard, 1966) responsivity. Increased and decreased reactivity have also been observed when subjects are told to attend to the stimuli (Barry, 1977; Korn \& Moyer, 1968; Hulstijn, 1978; Gliner, Harley, \& Badia, 1971; Maltzman \& Raskin, 1979; Pendergrass \& Kimmel, 1968), and some investigations have failed to uncover any effects of "attend" instructions (Badia \& Harley, 1970; Harding \& 
Punzo, 1971). A similarly confusing picture emerges when the effects of competing tasks on habituation are considered. Such tasks have been found to delay (Bohlin, 1976) and to speed (Frith \& Allen, 1983; Siddle, 1971) habituation. They have also been shown to have no effect (Frith \& Allen, 1983; Furedy, 1968; van Olst, Heemstra, \& ten Kortenaar, 1979).

Recently, we reported the results of a study contrasting the effects of different types of instructions and a distracting task on habituation to tones of various intensities (Iacono \& Lykken, 1983). Subjects were divided between two groups ("Ignore" and "Attend"), with subgroups of the subjects in each exposed to $70-, 90-$, or $110-\mathrm{dB}$ tones while they listened to a radio play. Subjects in the "Ignore" group were exhorted to immerse themselves in the story and ignore the tones. Subjects in the "Attend" group were instructed to count the tones and to be certain they all sounded the same. Another group ("Neutral"), exposed only to $110-\mathrm{dB}$ tones, received the neutral, ambiguous instructions common to habituation studies. The results indicated that the "Ignore" groups were less responsive and habituated faster than the "Attend" groups. These effects were especially pronounced at 70 and $110 \mathrm{~dB}$. When the "Neutral" group was compared with the others at $110 \mathrm{~dB}$, it was found to have response characteristics that were similar to those of the "Attend" group. This finding indicated that the specific instructions to attend to the stimuli did not augment responding and suggested that the faster habituation evident under the Ignore condition resulted from these subjects' understanding that the tones would be unchanging and could therefore safely be ignored.

Given the inconsistencies in the literature regarding the effects of instructions and competing tasks on habituation and the novel nature of our approach and results, we undertook a constructive replication (Lykken, 1968) and extension of our earlier findings. In the present investigation, we used a visual distractor, thereby providing an opportunity to determine the effects of instructions when the task and habituation stimuli involved different sensory systems. We also used two neutral groups, one with and the other without the distractor. The inclusion of the two neutral groups made it possible to determine the contribution of the distracting task to habituation, independent of the ignore or attend instructions.

\section{METHOD}

\section{Subjects}

Ninety-one undergraduate university students served as subjects. Three of these individuals were not included in the data analysis because they were taking medications or because the equipment failed. The remaining 88 subjects, half of whom were female, were evenly divided among the four experimental groups. The subjects ranged in age from 18 to $34(M=22.6, S D=3.9)$ years, and there were no significant differences in age across groups. All subjects gave informed consent and received course credit or were paid for their participation in the 1-h session.

\section{Apparatus and Recording Technique}

Skin conductance was recorded from Beckman 1-cm biopotential electrodes attached to the distal phalanges of the first and third fingers of each hand. The electrolyte consisted of physiological saline mixed with Unibase following the recipe provided by Fowles, Christie, Edelberg, Grings, Lykken, and Venables (1981). To ensure that the surface area of skin in contact with the electrolyte was similar for each finger and both hands, two electrode collars were used to attach each electrode. The first collar, which had a $0.7-\mathrm{cm}$ diameter, was attached to the subject's skin after the center of the collar was aligned with the center of the fingerprint. The second collar, which had a $1-\mathrm{cm}$ diameter, was affixed to the electrode. Then the two collars were aligned and the electrode was attached. This procedure guaranteed that there was no leakage between the electrode collar and the skin and that the surface area of skin in contact with the electrolyte was about $.38 \mathrm{~cm}^{2}$. Conductance was recorded with a Beckman Type RM Dynograph using two Type 9844 skin conductance couplers. Maximum sensitivity was 1 micromho/ $\mathrm{cm}$ of chart deflection.

Seventeen pure tones served as the habituation stimuli. All were $.5-\mathrm{sec}, 1000-\mathrm{Hz}$ tones with $40-\mathrm{msec}$ rise and fall times. These stimuli were delivered binaurally through AKG K240 stereo headphones at an intensity of $105 \mathrm{~dB}$ (re: $.0002 \mathrm{dyne} / \mathrm{cm}^{2}$ ). The tones were presented against a 50-dB pink-noise background and occurred at pseudorandom intervals ranging from 30 to $90 \mathrm{sec}$ and averaging $54 \mathrm{sec}$. Prior to the initiation of the tone series, all subjects received, without warning, a 2-sec, 105-dB pink-noise blast. This surprise stimulus was intended to elicit an estimate of the subject's maximum skin conductance response.

The visual distractor was an old silent movie. A videotape of the movie was played on a television monitor with a 19-in. diagonal screen. The monitor was positioned at eye level, approximately $1.5 \mathrm{~m}$ from the subject.

The two electrodermal channels were fed from the polygraph to a computer, which digitized these analog signals at a rate of $50 \mathrm{~Hz}$ for $20 \mathrm{sec}$ following stimulus onset. The computer also gave a trialby-trial Teletype printout of skin conductance response (SCR) amplitude for each hand.

\section{Procedure}

Upon reporting for the experiment, each volunteer was shown the apparatus and was asked to sign the consent form. The electrodes were then attached, and the subject was seated in a comfortable chair in a shielded audiometric chamber. All instructions were delivered via a tape recording.

To ensure that the experimental groups did not differ in electrodermal responsivity, the subjects were matched for SCR amplitude to the noise blast. This was accomplished by averaging the two SCRs elicited on each hand and assigning subjects such that the mean amplitude and variance of the SCR to the noise burst was balanced in the four groups. Approximately $2 \mathrm{~min}$ after the noise blast, the subjects heard one of the following sets of instructions:

Ignore (I) group. "You will be hearing some loud sounds during the next half-hour or so but from now on the sounds will be brief tones-rather than that blast of static you heard a few moments ago. We're going to measure how well you can ignore a series of distracting tones. These tones will be repeated at unpredictable in tervals; they will all be exactly alike and they will be meaninglessyou don't have to think about them or do anything when you hear them. Because the tones are loud, you will probably show a reaction to them at first; our instruments will be measuring your physiological reactions. But you will gradually get used to the tones and your body's response to each tone will get smaller and smaller. We want to measure your ability to "tune out" these meaningless tones, to see how quickly you can stop responding to them. Think of this as a kind of test; to do well on the test, you 
must ignore the tones. The best way to ignore the tones is to focus your attention on something else. Therefore, we're going to show you a movie on the TV set in front of you, an old silent film classic called "The Mark of Zorro," starring Douglas Fairbanks. Fix your attention on the movie, follow the action, think about what's going on in the film. We'll ask you a few questions about the movie when it's over to make sure that you paid close attention. Remember, by becoming absorbed in the movie, you will be best able to ignore the tones. While you're watching the movie, you'll hear a soft, rustling sound on the headphones. Remember: concentrate on the movie and try to ignore the tones. Find a comfortable position now and then try not to move until the movie is over. We'll turn the room light off now."

Attend $(A)$ group. "During the next half hour, you will be listening to a series of loud tones. The tones will all sound very much alike but some of them may differ slightly in some way from the others. We want you to pay close attention to these tones. Listen to them carefully and try to determine which ones are different and how they are different from the others. To keep track of what your're hearing, count the tones as they occur and try to remember the numbers of the tones that you think are different from the others. We'll ask you some questions later to see if you detected the differences. So that you won't get bored, we are going to play an old silent movie on the TV set in front of you. The film is called "The Mark of Zorro"' and stars Douglas Fairbanks. But don't get so absorbed in the movie that you forget to count the tones and remember which ones are distinctive. Find a comfortable position now and then try not to move until the session is over. When the movie ends, we'll turn on the room light and ask you some questions about the tones. We will turn off the room light now and then you'll hear a soft, rustling noise in the headphones."

Neutral-movie ( $N-M)$ group. "During the remainder of the session, we will be recording your body's responses while you listen to a series of brief, loud tones. You will hear the tones through the headphones you are wearing. You will also hear a soft, rustling noise over these headphones. So you won't get bored, we're going to show an old silent film on the TV screen in front of you. The film is called "The Mark of Zorro" and stars Douglas Fairbanks. Get into a comfortable position now and then try not to move until the session is over. When the movie ends, we'll turn on the room light and let you know that we're finished. We'll turn off the room light now."

Neutral ( $N$ ) group. "During the remainder of the session, we will be recording your body's responses while you listen to a series of brief, loud tones. You will hear the tones through the headphones you are wearing. You will also hear a soft, rustling noise over these headphones. Get into a comfortable position now, relax, and try not to move until the session is over. You will know when it's over when we turn on the room light again; the room light will come on about 20 seconds after the last tone in the series-if the light does not come on, that means that we haven't yet finished. We'll turn off the room light now."

Approximately 2 min after the movie began (or after the instructions to the neutral group were completed), the computer was activated and the tone series began about $30 \mathrm{sec}$ later. Twenty seconds after the last tone, the videotape monitor was shut off and the room light was turned on. The subjects were then asked to complete a questionnaire about the experimental session. They were asked how many tones had been presented and whether there had been a tone in the series that had differed from the others (if so, they were asked how it had differed from the others and where in the series it had occurred). Since all the tones were, in fact, identical, the questions about the "different" tone were included primarily to give the attend manipulation face validity and the responses were not used in the data analysis. The subjects also rated, on 7point scales, the loudness of the tones and how interesting they found the experiment. Those viewing the videotape were also asked to answer six questions about events that occurred in the movie. At the end of the session, the experimenter reviewed all the questions and answers with each subject to be certain they could be interpreted unambiguously. Although we had intended to eliminate I subjects who could not recall facts about the movie and $A$ subjects who did not count the tones, all the subjects in these groups exceeded our cutoff scores for performance on these variables.

Students viewing the videotape were also required to rate, on 7 point scales, their level of interest in the movie and their desire to see the remaining, unshown portion of the movie.

\section{Skin Conductance Measures}

SCR amplitude was measured as the difference between the maximum reached between 1 and $5 \mathrm{sec}$ of stimulus onset and the prestimulus level. All SCR data were range corrected (Lykken, 1972; Lykken, Rose, Luther, \& Maley, 1966) by dividing response amplitudes by each subject's maximum SCR. This was done separately for each hand. Ninety-six percent of the largest responses were elicited by the noise burst; the remainder were generated in response to the first tone.

In addition to analyses based on SCR amplitude, responsivity was indexed by counting the number of responses to the 17 tones, and rate of habituation was determined by tallying the number of trials preceding three consecutive nonresponses. For both of these measures, a response was counted if the SCR was 0.05 micromhos or larger in amplitude. Spontaneous responses were defined as phasic increases in conductance of at least 0.05 micromhos that occurred during the period from $10 \mathrm{sec}$ after each tone to the subsequent tone. Spontaneous responses were tallied for each quarter of the habituation run. Skin conductance level (SCL) was measured immediately prior to each tone.

\section{Statistical Procedures}

To reduce the number of false positives in the repeated measures analyses of variance (ANOVA) described below, the epsiloncorrection procedure described by Greenhouse and Geisser (1959; see also Keselman \& Rogan, 1980) was used to adjust the degrees of freedom. The F tests, which use repeated measures and are presented below, include the unadjusted degrees of freedom together with the value of epsilon associated with the error term and the corrected $\mathrm{p}$ value.

\section{RESULTS}

\section{Habituation Effects}

The phasic and tonic skin conductance data were analyzed with a four-way ANOVA, using groups (I, $A, N-M, N) \times$ sex (male, female) $\times$ hands (right, left) $x$ trials $(1-17)$ with repeated measures on the last two factors. The spontaneous response data were similarly analyzed, with quarters substituted for the trials factor. The amplitude of the response to the first tone, the number of tone-elicited responses, and the number of responses preceding three consecutive zero responses, were analyzed using a three-way ANOVA (omitting the trials factor).

Phasic responding. The range-corrected skin conductance response data for the four groups are plotted in Figure 1. An ANOVA applied to these data yielded significant main effects for groups $[F(3,80)$ $=3.04, \mathrm{p}<.05]$ and trials $[\mathrm{F}(16,1280)=38.91$, $\mathrm{p}<$ $.0001, \varepsilon=.48]$. Two interactions were significant, trials $\times$ group $[F(48,1280)=1.64, p<.05, \varepsilon=.48]$ and hand $x \operatorname{sex}[F(1,80)=5.12, p<.05]$. None of the other main or interaction effects attained significance. These results confirm the observations from 


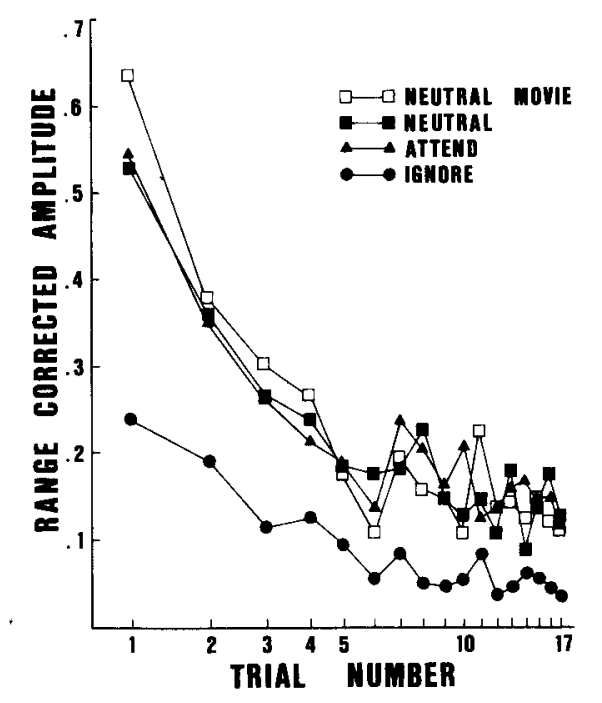

Figure 1. Range-corrected skin conductance amplitude as a function of trial number. The I (ignore) group was instructed to ignore the tone stimull and to watch a silent movie; the $A$ (attend) group was told to attend to the tones while the movie was presented; the $\mathbf{N}$ (neutral) group was given no specific instructions about the tones and no movie to watch; the $N-M$ (neutral-movie) group was given no specific instructions about the tones, and the movie was presented.

Figure 1 that: (1) subjects in the I group were less responsive than those in the other groups, (2) response amplitude diminished with trial repetition, and (3) the rate of response decrease over trials differed between the I subjects and those in the other three groups. The hand $\times$ sex interaction was attributable to female subjects' producing larger left-hand responses, whereas there was no difference between hands for males.

The amplitude of the first response was examined to determine whether the I group was initially less responsive than the others (see Figure 1). The results of a three-way ANOVA applied to these data revealed that this was indeed the case $[F(3,80)=9.13, p<$ $.0001]$. None of the other main or interaction effects attained statistical significance.

Figure 2 illustrates the number of trials preceding habituation for each group. An ANOVA applied to these data produced only a significant group effect $[F(3,80)=2.87, p<.05]$, confirming that the I subjects habituated at a faster rate than did subjects in the other groups. The I group also produced fewer SCRs to the tones than did the other groups $[F(3,80)$ $=2.79, \mathrm{p}<.05]$. Although the I group differed significantly from each of the other three groups on both of these variables, the A, N-M, and N groups did not differ among themselves.

Tonic skin conductance. The four-way ANOVA conducted on the SCL data failed to reveal any significant main effects for group, sex, or hand. The trials effect was, however, statistically significant
$[\mathrm{F}(16,1264)=15.96, \mathrm{p}<.0001, \varepsilon=.40]$ and confirmed that the tonic level of all groups dropped over time. Two interactions with trials were also significant. The trials $\times$ sex effect $[F(16,1264)=2.02, p<$ $.01, \varepsilon=.15]$ indicated that SCL declined at a faster rate for females than for males. The interaction with hands $[F(16,1264)=3.89, p<.05, \varepsilon=.13]$ revealed that the difference in tonic conductance between hands (SCL was higher on the right side than on the left) was reduced with successive tone presentations.

Spontaneous responding. Analysis of the spontaneous response data produced only one significant effect, a three-way interaction between quarters, groups, and $\operatorname{sex}[F(9,240)=2.02, p<.05, \varepsilon=.88]$. This result was due primarily to the males in the N-M group, who showed a marked increase in the number of spontaneous responses over quarters, whereas the remaining subjects showed a trend indicating a small decrease in activity over time.

\section{Questionnaire Data}

One-way ANOVAs were used to determine if there were group differences in the number of tones subjects reported hearing, the perceived loudness of the tones, and the level of interest in the experiment. Only the ratings of tone loudness produced a significant effect $[F(3,84)=6.20, p<.001]$. A posteriori contrasts confirmed the observation from Figure 3 that the Ignore subjects perceived the tones as less intense than did subjects in each of the other groups.

Additional one-way ANOVAs were computed for subjects viewing the videotape to determine if the $I$, $A$, and N-M groups differed in the number of ques-

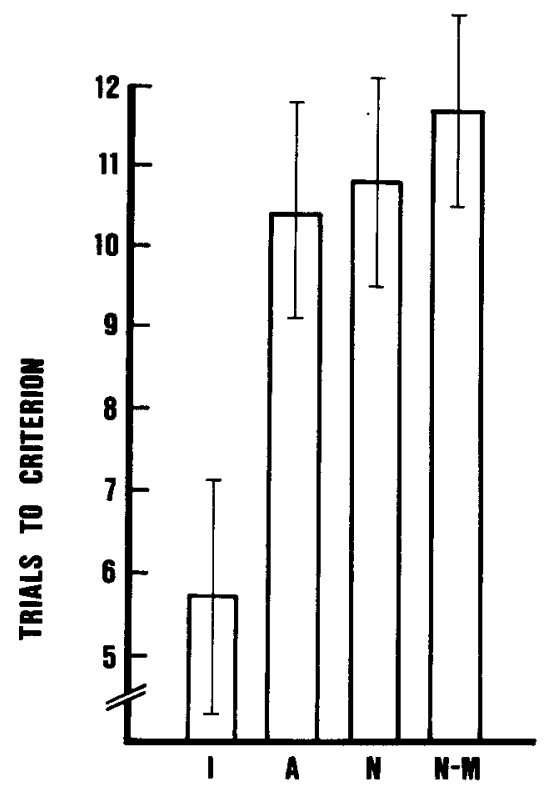

Figure 2. The mean \pm the standard error for the number of trials to three consecutive zero responses. I = ignore group, $A=$ attend group, $\mathbf{N}=$ neutral group, $\mathbf{N}-\mathbf{M}=$ neutral-movle group. See the caption to Figure 1 for a description of the groups. 


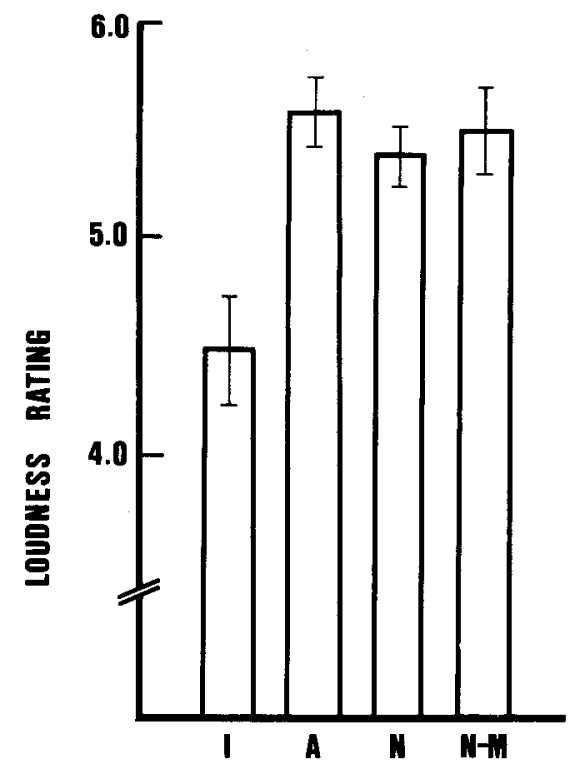

Figure 3. The mean \pm the standard error ratings of stimulus intensity. I = ignore group, $\mathbf{A}=$ attend group, $\mathbf{N}=$ neutral group, $\mathbf{N}-\mathbf{M}=$ neutral-movie group. See the caption to Figure 1 for a description of the groups.

tions about the movie answered correctly, in the desire to see the conclusion of the movie, and in the level of interest in the movie. No significant group differences were found for these variables.

\section{DISCUSSION}

The results clearly indicate that instructions to ignore the stimuli coupled with an engaging visual task affect electrodermal responsivity. The I subjects were less responsive and habituated at a faster rate than did their counterparts in the other groups. They also rated the tones as less loud than the other subjects, a finding that suggests that the psychological impact of the tones was reduced for the I group.

In our earlier study, I subjects received both special instructions and a distracting task, whereas those in the $\mathbf{N}$ group were given neither. Hence, the relative contribution of each of these two factors to the results was unclear. The use of two neutral groups in our present study, only one of which viewed the movie, sheds some light on this issue. The I subjects were less responsive than those in either neutral group, and the two neutral groups did not differ in their response characteristics. These findings indicate that the presence of the distractor in the I condition cannot account for the observed results. Hence, the additional task instructions the I group received were critical to obtaining group differences in electrodermal responding.

These instructions differed in two ways from those used for the neutral groups: The subjects were told to ignore the tones and to focus their attention on the movie. Either of these manipulations could account for the results. That paying attention to the movie may contribute to reduced responding is suggested by the work of Maltzman, Vincent, and Wolff (1982). Those investigators found that when attention was directed to one stimulus in a series, by requiring a pedalpress to its occurrence, the response to a subsequently presented stimulus was diminished as compared with the responses generated by the same stimuli without the pedalpress requirement. Maltzman et al. (1982) hypothesized that "a voluntary and effortful focusing of attention towards one event narrows the focus of attention and as a consequence decreases the apprehension of other stimuli" (p. 226). A direct test of this notion in the present study would require another control group in which ignore instructions were omitted but subjects were exhorted to attend to the movie. However, our data indicate that even in the absence of specific instructions to attend to the movie, subjects in the N-M group did so anyway. There were no differences between groups in the number of questions about the movie answered correctly, in the level of interest expressed in the movie, or in the desire to watch the movie to its conclusion. To the extent that these results indicate that both the I and N-M groups attended to the movie, it seems likely that the critical difference between these groups was the instructions indicating that the stimulus was meaningless and should be ignored.

Even though the A subjects in our previous study were given instructions to enhance the signal value of the tones, their pattern of electrodermal response did not differ from that of the $\mathrm{N}$ group. Subjects in both groups responded an average of 16 times to the 17 tones. However, the limit on the number of trials imposed an obvious ceiling that may have prevented differences in responsivity and habituation rate from emerging. In our present study, the ceiling effect was not a problem, perhaps because we used less intense tones and electrode contact areas that were about half as large as those used previously (although cf. Mitchell \& Venables, 1980). The A group met our habituation criterion in under 11 trials, and the two neutral groups actually habituated at a marginally slower rate. Hence, the attend instructions did not affect responsivity. Although there are some studies indicating that instructions to pay attention to tonal stimuli are not associated with increased responding (Barry, 1977; Harding \& Punzo, 1971), other investigations have found that such instructions increase responding and/or delay habituation (Hulstijn, 1978; Korn \& Moyer, 1968; Pendergrass \& Kimmel, 1968). However, the strongest stimuli used in any of these studies had an intensity of $90 \mathrm{~dB}$ (Pendergrass \& Kimmel, 1968), well below that used in our two studies. It could be that with very loud tones, such as 
those we have used, the effects of the stimuli are so overwhelming that paying special attention to them does not augment their psychological impact.

The trials-to-criterion measure of habituation indicated that in both studies the I group stopped responding earlier than the other groups. These results could mean either that response inhibition with stimulus repetition proceeds at a faster rate in the Ignore condition or that the I subjects habituate faster because they are less responsive to begin with. The results of both studies favor the latter interpretation and suggest that Ignore subjects activate a gating mechanism that attenuates the effect of the stimulus. This conclusion is supported by the present findings that the intensity of the tones was rated as less by the I group than by other groups. It is also buttressed by the result from the previous study, which indicated that, in estimating the number of tones to which they had been exposed, the I subjects estimated fewer than did the other subjects. The responsivity data directly support this interpretation. I subjects in both studies consistently produced smaller SCRs to the stimuli. Moreover, in the present study, the response to the first tone was smaller in the I group, and the significant trials $\times$ group interaction coupled with inspection of Figure 1 reveals that the rate of response decrement was highest in the $\mathbf{A}$ and two neutral groups.

The subjects in the neutral groups, who received the vague instructions common to many habituation studies, were more responsive to the tones than were the I subjects, and, furthermore, they did not differ from the subjects in the A group. These results suggest that the significance of the tones did not differ for the members of the two neutral and A groups. Because the instructions to subjects in the A group required them to give the tones signal value, the lack of difference between the two neutral and A groups suggests that "neutral" instructions may actually retard habituation by allowing the stimuli to acquire significance. This conclusion is consistent with our belief that "neutral" instructions are not well suited to habituation studies in which the objective is to measure an individual's ability to tune out and stop reacting to a meaningless stimulus.

There were several failures to replicate some results from our first study. One of the interesting findings from our other investigation was that although tonic conductance habituated for the I group, a pattern of sensitization was evident in the $A$ and neutral groups. These data are consistent with the dual process theory of Groves and Thompson (1970), which hypothesizes that habituation and sensitization are independent phenomena that interact to produce the final response pattern. Groves and Thompson speculate that sensitization is a function of stimulus intensity and the subject's "state," which, in this case, may be indexed by tonic skin conductance. In light of the dual process theory, we interpreted our findings as indicating that the ignore instructions had a major effect on the state system. However, in the present study, all groups showed SCL habituation.

There were several differences between the two studies that could account for these discrepant findings. The tones used in the present study were not as loud as before and therefore might not have been strong enough to elicit a sensitization response. In the present study, a loud burst of white noise preceded the habituation run. This stimulus may have triggered a sensitization response that waned prior to the presentation of the first tone. Data from the first study support this notion; the large increase in SCL indicative of possible sensitization occurred after the first stimulus presentation. Finally, the nature of the distracting task could account for the differences. Focusing on a visual, rather than auditory, distractor ${ }^{\text {. }}$ may have made it easier to attenuate the impact of the loud tones on the state system.

Another difference between the studies concerns the questionnaire responses. I subjects in the first study estimated that there were significantly fewer tones than did subjects in other groups. While the trend in the data was the same in the present study, the differences between groups were not significant. Although there were no significant differences between groups in ratings of tone loudness in our earlier study, differences in the expected direction emerged in our present investigation. While any of the differences between the two studies discussed in the preceding paragraph could be responsible for these outcomes, perhaps the one most likely to account for these discordant results involves the use of distracting tasks in different sensory modalities. It may be difficult to ignore the loudness of a stimulus when one is absorbed in a task requiring auditory attention and information processing. On the other hand, a visually engaging task plus the ignore instructions provide an opportunity to "tune out" auditory stimuli altogether.

In conclusion, the results from both studies are congruent in showing that instructions to ignore the stimuli coupled with a distracting task reduce responding to loud stimuli. These findings are consistent with recent modifications of OR theory that indicate the subject's judgments of stimulus significance and expectations or "set" regarding the stimuli affect the orienting response and habituation (Bernstein, 1979; Maltzman, 1979).

\section{REFERENCES}

Badia, P., \& Harley, J. P. (1970). Habituation and temporal conditioning as related to shock intensity and its judgment. Journal of Experimental Psychology, 84, 534-536.

BARRY, R. J. (1977). The effect of "significance" upon indices of Sokolov's orienting response: A new conceptualization to replace the OR. Physiological Psychology, 5, 209-214. 
Bernstein, A. S. (1979). The orienting response as novelty and significance detector: Reply to O'Gorman. Psychophysiology, 16, 263-273.

Bernste in, A. S., Frith, C. D., Gruzelier, J. H., Straube, E. S., Venables, P. H., \& ZahN, T. P. (1982). An analysis of skin conductance orienting response in samples of American, British, and German schizophrenics. Biological Psychology, 14, 155-211.

BoHLiN, G. (1976). Delayed habituation of the electrodermal response as a function of increased level of arousal. Psychophysiology, 13, 345-351.

Fowles, D. C., Christie, M. J., Edelberg, R., Grings, W. W., Lykken, D. T., \& Venables, P. H. (1981). Publication recommendations for electrodermal measurements. Psychophysiology, 18, 232-239.

Frith, C. D., \& Allen, H. A. (1983). The skin conductance orienting response as an index of attention. Biological Psychology, 17, 27-40.

FUREDY, J. J. (1968). Human orienting reaction as a function of electrodermal versus plethysmographic response modes and single versus alternating stimulus series. Journal of Experimental Psychology, 77, 70-78.

Gliner, J. A., Harley, J. P., \& Badia, P. (1971). Elicitation and habituation of the orienting response as a function of instructions, order of stimulus presentation, and omission. Journal of Experimental Psychology, 89, 414-416.

Greenhouse, S. W., \& Geiser, S. (1959). On methods in the analysis of profile data. Psychometrika, 24, 95-112.

Groves, P. M., \& Thompson, R. H. (1970). Habituation: A dualprocess theory. Psychological Review, 77, 419-450.

Harding, G., \& Punzo, F. (1971). Response uncertainty and skin conductance. Journal of Experimental Psychology, 88, 265-272.

Hulstijn, W. (1978). Habituation of the orienting response as a function of arousal induced by three different tasks. Biological Psychology, 7, 109-124.

Iacono, W. G., \& Lykken, D. T. (1979). The orienting response: Importance of instructions. Schizophrenia Bulletin, 5, 11-14.

IACono, W. G., \& LykKen, D. T. (1983). The effects of instructions on electrodermal habituation. Psychophysiology, 20, $71-80$.

Iacono, W. G., Lykken, D. T., Peloquin, L. J., Lumry, A. E., Valentine, R. H., \& Tuason, V. B. (1983). Electrodermal activity in euthymic unipolar and bipolar affective disorders: A possible marker for depression. Archives of General Psychiatry, $40,557-565$.

Keselman, H. J., \& Rogan, J. C. (1980). Repeated measures F tests and psychophysiological research: Controlling the number of false positives. Psychophysiology, 17, 499-503.

Kimmel, H. D., van Olst, E. H., \& Orlebeke, J. F. (Eds.) (1979). The orienting reflex in humans. Hillsdale, NJ: Erlbaum.

KoHLENBERG, R. J. (1970). Instructions to ignore a stimulus and the GSR. Psychonomic Science, 19, 220-221.

Korn, J. H., \& MoYer, K. E. (1968). Effects of set and sex on the electrodermal orienting response. Psychophysiology, 4, 453-459.

LYKKEN, D. T. (1968). Statistical significance in psychological research. Psychological Bulletin, 70, 151-159.

LYKKEN, D. T. (1972). Range correction applied to heart rate and to GSR data. Psychophysiology, 9, 373-379.

Lykken, D. T., Rose, R., Luther, B., \& Maley, M. (1966). Correcting psychophysiological measures for individual differences in range. Psychological Bulletin, 66, 481-484.

Maltzman, I. (1979). Orienting reflexes and significance: A reply to O'Gorman. Psychophysiology, 16, 274-292.

Maltzman, I., \& Raskin, D. C. (1979). Selective orienting and habituation of the GSR as a consequence of overt and covert activity. Physiological Psychology, 7, 204-208.

Maltzman, I., Vincent, C., \& WolfF, C. (1982). Verbal conditioning, task instructions, and inhibition of the GSR measure of the orienting reflex. Physiological Psychology, 10, 221-228.

Mitchell, D. A., \& Venables, P. H. (1980). The relationship of EDA to electrode size. Psychophysiology, 17, 408-412.

O'GonmaN, J. G. (1977). Individual differences in habituation of human physiological responses: A review of theory, method, and findings in the study of personality correlates in nonclinical populations. Biological Psychology, 5, 257-318.

Pendergrass, V. E., \& Kimmel, H. D. (1968). UCR diminution in temporal conditioning and habituation. Journal of $E x$ perimental Psychology, 77, 1-6.

Sidple, D. A. T. (1971). The orienting response and distraction. Australian Journal of Psychology, 23, 261-265.

Tizard, B. (1966). Evoked changes in EEG and electrodermal activity during the waking and sleeping states. Electroencephalography \& Clinical Neurophysiology, 20, 122-128.

van Olst, E. H., Heemstra, M. L., \& ten Kortenaar, T. (1979). Stimulus significance and the orienting reaction. In H. D. Kimmel, E. H. van Olst, \& J. F. Orlebeke (Eds.), The orienting reflex in humans. Hillsdale, NJ: Erlbaum.

(Manuscript received October 11, 1983; revision accepted for publication February 24, 1984.) 